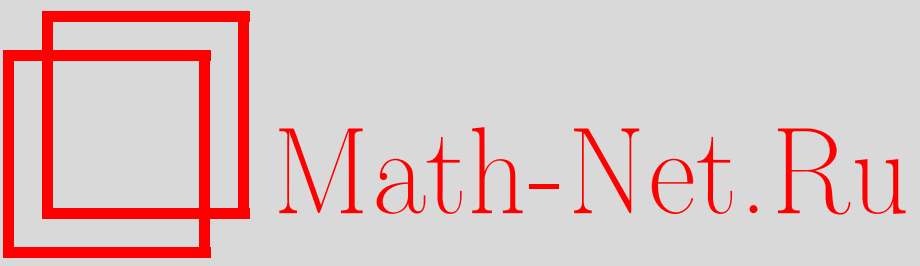

О. П. Соловцова, Теория возмущений и аналитический подход применительно к инклюзивному распаду $\tau$-лептона, ТМФ, 2003, том 134, номер 3, 416-429

DOI: https://doi.org/10.4213/tmf168

Использование Общероссийского математического портала Math-Net.Ru подразумевает, что вы прочитали и согласны с пользовательским соглашением

http://www . mathnet.ru/rus/agreement

Параметры загрузки:

IP : 35.173 .219 .12

26 апреля 2023 г., 13:02:08 
ТЕОРЕТИЧЕСКАЯ

И МАТЕМАТИЧЕСКАЯ

ФИЗИКА

Том 134, № 3

март, 2003

(C) 2003 r.

О. П. Соловцова*

\section{ТЕОРИЯ ВОЗМУЩЕНИЙ И АНАЛИТИЧЕСКИЙ ПОДХОД ПРИМЕНИТЕЛЬНО К ИНКЛЮЗИВНОМУ РАСПАДУ $\tau$-ЛЕПТОНА}

Выполнен сравнительный анализ различных форм пертурбативных разложений в пространственноподобной и времениподобной областях. Применительно к инклюзивному распаду $\tau$-лептона дано сравнение результатов, полученных при использовании стандартной теории возмущений и аналитического подхода Ширкова-Соловцова, который модифицирует пертурбативные разложения таким образом, чтобы новые аппроксимации отражали основные принципы теории, такие как ренормализационная инвариантность, спектральность и причинность. Показаны преимущества и самосогласованность аналитического подхода при описании распада $\tau$-лептона.

Ключевые слова: квантовая хромодинамика, ренормализационная группа, теория возмущений, $\tau$-лептон.

\section{1. ВВЕДЕНИЕ}

Теория возмущений (ТВ) является одним из основных инструментов проведения вычислений в квантовой теории поля [1]. Успехи ТВ в сочетании с процедурой перенормировок и методом ренормализационной группы (РГ) в квантовой электродинамике, в теории электрослабых взаимодействий и в пертурбативной области квантовой хромодинамики (КХД) неоспоримы. При описании адронных процессов, для которых характерный энергетический масштаб принадлежит низкоэнергетической области, ограничиться лишь пертурбативным анализом нельзя. Здесь важную роль играют непертурбативные эффекты, которые следует учитывать наряду с ТВ-вкладами. Таким образом, в теоретическом описании присутствуют связанные друг с другом пертурбативная и непертурбативная компоненты. Это приводит к тому, что неопределенность, вызванная неизбежным обрывом ряда ТВ, оказывает влияние на надежность информации о непертурбативных поправках. В КХД, благодаря свойству асимптотической свободы, пертурбативная компонента доминирует при больших энергетических масштабах, так как в этом случае неизвестные вклады высших порядков ТВ малы. Однако высокоэнергетическая область, в которой характерный масштаб процесса составляет десят-

* Объединенный институт ядерных исследований, Дубна, Московская обл., Россия. Е-mail: olsol@thsun1.jinr.ru 
ки ГэВ, малоинформативна с точки зрения исследования сушественно непертурбативных эффектов. Для изучения низкоэнергетической области возникает необходимость в разработке методов, позволяющих модифицировать пертурбативные разложения с целью снижения теоретических неопределенностей, связанных, в частности, с зависимостью результатов от выбора ренормализационного предписания. Здесь важным оказывается тот факт, что исходный ряд ТВ, точнее, его конечная часть после выполнения процедуры перенормировки, не является конечным продуктом теории, а допускает значительную модификацию. Так, например, хорошо известно, что применение метода РГ [2]-[4] позволяет модифицировать пертурбативное разложение в соответствии с общим принципом ренормализационной инвариантности, улучшив при этом свойства ряда в ультрафиолетовой области. Что касается инфракрасной области, в которой пертурбативный инвариантный заряд обладает нефизическими сингулярностями (в однопетлевом приближении - это хорошо известный “призрачный полюс" ), то применение РГ-модифицированного ряда ТВ не позволяет получать стабильные результаты.

В работе [5] в контексте квантовой электродинамики был предложен способ устранения нефизических особенностей у инвариантного заряда. Недавно эта идея была применена для случая КХД [6]. Развитый в этих работах аналитический подход объединяет метод РГ и $Q^{2}$-аналитичность, отражающую общие принципы локальной квантовой теории поля, такие как спектральность и причинность, и приводит к ряду новых интересных свойств разложений [7]. Дальнейшему развитию и применению аналитического метода посвящены многие работы, среди которых отметим [8]-[15]. Так, например, было установлено, что в рамках аналитического подхода удается непротиворечивым образом определить эффективный заряд во времениподобной области [8], что дает возможность самосогласованного описания в рамках этого подхода процессов с характерными пространственноподобными $\left(q^{2}<0\right)$ и времениподобными $\left(q^{2}>0\right)$ импульсами [9]. Сформулированная в работе [10] аналитическая теория возмушений (АТВ) обеспечивает правильные аналитические свойства таких важных объектов как, например, коррелятор токов и обладает слабой чувствительностью к выбору схемы перенормировок. Теоретическая неопределенность в АТВ, связанная с конечностью порядка аппроксимации, сушественно уменьшается. Использование АТВ в качестве пертурбативной составляющей КХД-анализа влияет на величину получаемых из экспериментальных данных непертурбативных параметров. Например, выполненный в [13] анализ правила сумм Гросса-Ллевеллина Смита показал, что $Q^{2}$-эволюция, полученная в ТВ без учета вклада высших твистов, практически совпадает с результатом АТВ-анализа, но с учетом поправки $1 / Q^{2}[16]$, оцененной в [17] методом правил сумм КХД.

Цель данной работы состоит в изучении особенностей применения ТВ- и ATB-разложений при описании инклюзивного распада $\tau$-лептона в адроны. Открытый еше в 1975 году, $\tau$-лептон является единственным известным лептоном, масса которого $M_{\tau} \simeq$ 1, 78 ГэВ, с одной стороны, достаточно велика, чтобы могли наблюдаться адронные моды распада, а с другой стороны, в шкале КХД принадлежит области низких энергий. Экспериментальные данные по распаду $\tau$-лептона в адроны, полученные с рекордной для адронных процессов точностью [18]-[20], дают уникальную возможность для низ- 
коэнергетического тестирования КХД.

Далее в разделе 2 представлены основные соотношения. В разделе 3 дан анализ достоинств и недостатков стандартных ТВ-разложений. Аналогичное рассмотрение, выполненное в рамках АТВ-подхода, представлено в разделе 4. Теоретический анализ ТВи AТВ-разложений, возникающих при описании инклюзивного распада $\tau$-лептона в адроны, приведен в разделе 5, численные сравнения - в разделе 6 . Результаты анализа обсуждаются в заключении.

\section{2. ОСНОВНЫЕ СООТНОШЕНИЯ}

Описание инклюзивного распада $\tau$-лептона связано с коррелятором векторного и аксиально-векторного кварковых токов [21], [22],

$$
\Pi_{\mu \nu}\left(q^{2}\right)=i \int d^{4} x e^{i q x}\left\langle 0\left|T J_{\mu}(x) J_{\nu}(0)^{+}\right| 0\right\rangle \sim\left(q_{\mu} q_{\nu}-g_{\mu \nu} q^{2}\right) \Pi\left(q^{2}\right)
$$

Удобно использовать РГ-инвариантную функцию Адлера [23], связанную с коррелятором $\Pi\left(q^{2}\right)$ соотношением

$$
D\left(Q^{2}\right)=-q^{2} \frac{d \Pi\left(q^{2}\right)}{d q^{2}}
$$

Здесь мы используем обшепринятое соглашение $Q^{2}=-q^{2}>0$ в евклидовой области. Интегральное представление для $D$-функции записывается как

$$
D\left(Q^{2}\right)=Q^{2} \int_{0}^{\infty} \frac{d s}{\left(s+Q^{2}\right)^{2}} R(s),
$$

где $R(s)=\operatorname{Im} \Pi(s+i 0) / \pi$. Представление $(3)$ определяет функцию $D\left(Q^{2}\right)$ как аналитическую функцию в комплексной $Q^{2}$-плоскости с разрезом вдоль отрицательной части вещественной оси.

Определим КХД-вклады $d\left(Q^{2}\right)$ и $r(s)$ в функции $D \sim 1+d$ и $R \sim 1+r$, соответственно, которые связаны между собой соотношениями

$$
\begin{aligned}
d\left(Q^{2}\right) & =Q^{2} \int_{0}^{\infty} \frac{d s}{\left(s+Q^{2}\right)^{2}} r(s), \\
r(s) & =-\frac{1}{2 \pi i} \int_{s-i \epsilon}^{s+i \epsilon} \frac{d z}{z} d(-z) .
\end{aligned}
$$

Контур интегрирования в последней формуле расположен в области аналитичности подынтегрального выражения и обходит разрез на вешественной полуоси.

Выражения (4) и (5) могут быть переписаны через эффективную спектральную функцию $\rho^{\mathrm{eff}}(\sigma)[8]$,

$$
\begin{aligned}
d\left(Q^{2}\right) & =\frac{1}{\pi} \int_{0}^{\infty} d \sigma \frac{\rho^{\mathrm{eff}}(\sigma)}{\sigma+Q^{2}}, \\
r(s) & =\frac{1}{\pi} \int_{s}^{\infty} \frac{d \sigma}{\sigma} \rho^{\mathrm{eff}}(\sigma),
\end{aligned}
$$


которая определяется через скачок $d\left(Q^{2}\right)$ на разрезе.

Экспериментально измеряемое отношение адронной и лептонной ширин распада $\tau$ лептона можно представить в виде

$$
R_{\tau}=3\left(\left|V_{u d}\right|^{2}+\left|V_{u s}\right|^{2}\right) S_{\mathrm{EW}}\left(1+\delta_{\tau}\right),
$$

где $V_{u d}$ и $V_{u s}$ - элементы матрицы Кабиббо-Кобаяши-Маскава, а $S_{\mathrm{EW}}$ - известный электрослабый фактор [22]. Интересуюший нас вклад сильных взаимодействий $\delta_{\tau}$ выражается через $r(s)$ как

$$
\delta_{\tau}=2 \int_{0}^{M_{\tau}^{2}} \frac{d s}{M_{\tau}^{2}}\left(1-\frac{s}{M_{\tau}^{2}}\right)^{2}\left(1+2 \frac{s}{M_{\tau}^{2}}\right) r(s) .
$$

Соотношения (4) и $(5)$ позволяют записать $\delta_{\tau}$ в виде интеграла по окружности радиуса $M_{\tau}^{2}[24]$,

$$
\delta_{\tau}=\frac{1}{2 \pi i} \oint_{|z|=M_{\tau}^{2}} \frac{d z}{z}\left(1-\frac{z}{M_{\tau}^{2}}\right)^{3}\left(1+\frac{z}{M_{\tau}^{2}}\right) d(-z) .
$$

Легко также переписать $\delta_{\tau}$ через спектральные моменты,

$$
\delta_{\tau}=2 \mathrm{~m}_{0}\left(M_{\tau}^{2}\right)-2 \mathrm{~m}_{2}\left(M_{\tau}^{2}\right)+\mathrm{m}_{3}\left(M_{\tau}^{2}\right),
$$

где

$$
\mathrm{m}_{k}\left(s_{0}\right) \equiv(k+1) \int_{0}^{s_{0}} \frac{d s}{s_{0}}\left(\frac{s}{s_{0}}\right)^{k} r(s),
$$

или при использовании формулы (5)

$$
\mathrm{m}_{k}\left(s_{0}\right)=\frac{1}{2 \pi i} \oint_{|z|=s_{0}} \frac{d z}{z}\left[1-\left(\frac{z}{s_{0}}\right)^{k+1}\right] d(-z) .
$$

Понятно, что выражения (8) и (9), так же как (11) и (12), эквивалентны при условии наличия у функции $d(-z)$ упомянутых выше аналитических свойств.

\section{3. ТЕОРИЯ ВОЗМУШЕНИЙ}

$N$-й порядок теории возмушений для функций $r(s)$ и $d\left(Q^{2}\right)$ в случае безмассовых кварков имеет вид

$$
\begin{aligned}
d^{(N)}\left(Q^{2}\right) & =\sum_{n=1}^{N} a^{n}\left(\mu^{2}\right) \sum_{k=0}^{n} d_{n, k} \ln ^{k}\left(\frac{Q^{2}}{\mu^{2}}\right), \\
r^{(N)}(s) & =\sum_{n=1}^{N} a^{n}\left(\mu^{2}\right) \sum_{k=0}^{n} r_{n, k} \ln ^{k}\left(\frac{s}{\mu^{2}}\right) .
\end{aligned}
$$


Воспользовавшись соотношениями (4) и (5), получаем связь между коэффициентами

$$
\begin{aligned}
d_{n, k} & =\sum_{m=0}^{n-k} \frac{(k+m) !}{k ! m !} r_{n, k+m} J_{m} \\
r_{n, k} & =\sum_{m=0}^{n-k} \frac{(k+m) !}{k ! m !} d_{n, k+m} I_{m}
\end{aligned}
$$

где $J_{m}=I_{m}=0$ для нечетных $m$ и

$$
\begin{aligned}
J_{m} & =2 m ! \zeta(m)\left(1-2^{1-m}\right), \\
I_{m} & =(-1)^{m / 2} \frac{\pi^{m}}{m+1}
\end{aligned}
$$

для четных $m$. Здесь $\zeta(m)-\zeta$-функция Римана.

Различие коэффициентов $d_{n, k}$ и $r_{n, k}$ начинается с трехпетлевого уровня и связано с так называемыми $\pi^{2}$-членами. Так, например,

$$
d_{3,0}=r_{3,0}+r_{3,2} \frac{\pi^{2}}{3}=r_{3,0}+\beta_{0}^{2} \frac{\pi^{2}}{3}
$$

где $\beta_{0}=\left(11-2 n_{f} / 3\right) / 4$ - однопетлевой коэффициент ТВ-разложения $\beta$-функции. Такая обычно используемая " $\pi^{2}$-терминология" находится в соответствии с выражениями (17) и (18), если принять во внимание ${ }^{1)}$, что $\zeta(2 m) \sim\left(\pi^{2}\right)^{m}$. Здесь следует отметить, что не все функции $\zeta(2 m)$, появляюшиеся в рядах ТВ, связаны с эффектами аналитического продолжения. Например, значение функции $\zeta(4)$ возникает при вычислении поправки порядка $\alpha_{S}^{3}$ к коррелятору скалярных кварковых токов как в евклидовой области, так и при учете эффектов аналитического продолжения [30].

Далее, вклад $\pi^{2}$-членов, отличающих третьи пертурбативные коэффициенты в разложении "пространственноподобных" и “времениподобных" объектов, может привести к различным знакам второго схемного инварианта [31] для этих объектов. Это имеет место, например, для процесса $\left(e^{+} e^{-}\right)$-аннигиляции в адроны. В этом случае знаки второго инварианта для "времениподобного" отношения $R_{e^{+}} e^{-}$и "пространственноподобной” $D$-функции различны. Отрицательный знак второго схемного инварианта для $R_{e^{+} e^{-}}$ведет к инфракрасной заморозке эффективного заряда, которая возникает при оптимизации схемной зависимости [32], [33] на основе принципа минимальной чувствительности (PMS) [31] или метода эффективного заряда (ЕСН-метод) [34], [35]. В то же время знак второго схемного инварианта для $D$-функции остается положительным и

\footnotetext{
1) $\pi^{2}$-Вклады могут также быть получены в результате аналитического продолжения бегущей константы связи из евклидовой области в физическую [25]-[27]. Их роль обсуждалась, например, в работах [15], [28], [29].
} 
пертурбативный анализ, основанный как на PMS-, так и на ЕCH-оптимизации, не приводит к заморозке заряда в евклидовой области (см. обсуждение в работах [36], [37]). Тогда возникает несоответствие между результатом прямого применения PMS/ECH-оптимизаций к $D$-функции, приводящего к сингулярному инфракрасному поведению, и результатом вычисления этой функции через дисперсионное соотношение (3) (с использованием оптимизированной функции $R(s)$ ), что дает регулярную функцию на всем интервале $Q^{2}$. Здесь следует отметить, что результаты работ [32], [33], [36] не означают, что в действительности реализуется режим инфракрасной заморозки эффективного заряда. Этот режим лиш соответствует наличию нетривиального инфракрасного нуля у трехпетлевой эффективной $\beta$-функции. Поэтому высшие поправки могут полностью изменить ситуацию. Этот эффект был обнаружен в работе [38] для явно вычисленной четырехпетлевой эффективной $\beta$-функции.

Исходный ряд (13) обладает важным свойством: любой его член конечного порядка сохраняет правильные аналитические свойства $d$-функции. В то же время этот ряд имеет и негативные черты. Так, например, имеющийся произвол в выборе численного значения точки нормировки $\mu^{2}$ делает его плохо приспособленным для практических применений. K счастью, в квантовой теории поля исходные разложения типа (13) не являются конечным результатом теории, а допускают возможность сушественной модификации, которая может быть выполнена с учетом общих принципов теории. Так, метод РГ, аккумулируя большие логарифмы в инвариантный заряд, позволяет устранить $\mu$-зависимость пертурбативного ряда и улучшить его поведение в ультрафиолетовой области. Разложение по инвариантному заряду $\bar{a}\left(Q^{2}\right)$ имеет вид

$$
d^{(N)}\left(Q^{2}\right)=\sum_{n=1}^{N} d_{n} \bar{a}^{n}\left(Q^{2}\right),
$$

где $d_{n-1} \equiv d_{n, 0}$.

Таким образом, общий принцип ренормализационной инвариантности позволяет улучшить ультрафиолетовое поведение ряда ТВ и избавиться от $\mu$-зависимости в любом конечном порядке. Однако получаемые ряды по-прежнему остаются плохо определенными в инфракрасной области и, кроме того, утрачивается положительное свойство исходного разложения (13), так как теперь правильные аналитические свойства ряда в комплексной $Q^{2}$-плоскости теряются из-за нефизических особенностей $\bar{a}\left(Q^{2}\right)$. Тем самым нарушена связь между функциями $d\left(Q^{2}\right)$ и $r(s)$, задаваемая соотношениями (4) и (5). Так, если использовать в выражении (5) $d$-функцию в виде (20) и найденную таким способом функцию $r(s)$ подставить в (4), то первоначальная функция $d\left(Q^{2}\right)$, которая использовалась при получении $r(s)$, не воспроизведется. Более того, интеграл в (4) окажется расходящимся из-за нефизических особенностей $\bar{a}\left(Q^{2}\right)$.

Параметризация $r(s)$ в виде разложения по пертурбативному инвариантному заряду приводит также к проблеме вычисления величины $\delta_{\tau}$, определенной в $(8)$, поскольку в этом выражении интервал интегрирования включает в себя инфракрасную область. Казалось бы, что переход к контурному представлению (9) позволяет избежать возникшую трудность, так как нефизические особенности инвариантного заряда в этом случае лежат вне контура и процедура интегрирования может быть формально выполнена. 
Однако, по нашему мнению, этот прием ни в коей мере не решает проблемы, так как неправильные аналитические свойства инвариантного заряда приводят к тому, что выражения (8) и $(9)$ для $\delta_{\tau}$ более не эквивалентны. Например, в основном порядке для моментов (11) при $s_{0}>\Lambda^{2}$ находим

$$
\begin{aligned}
& \frac{1}{2 \pi i} \oint_{|z|=s_{0}} \frac{d z}{z}\left[1-\left(\frac{z}{s_{0}}\right)^{k+1}\right] \frac{1}{\ln \left(-\frac{z}{\Lambda^{2}}\right)}= \\
& \quad=(k+1) \int_{0}^{s_{0}} \frac{d s}{s_{0}}\left(\frac{s}{s_{0}}\right)^{k}\left[\frac{1}{2}-\frac{1}{\pi} \operatorname{arctg} \frac{\ln \left(\frac{s}{\Lambda^{2}}\right)}{\pi}+(-1)^{k} \Theta\left(\Lambda^{2}-s\right)\right] .
\end{aligned}
$$

Это выражение показывает, что, используя пертурбативный инвариантный заряд в контурном представлении (12), нельзя воспроизвести какой-либо аналог инвариантного заряда в начальном интеграле, поскольку выражение в квадратных скобках в правой части (21) содержит $k$-зависимость, которая, естественно, у инвариантного заряда должна отсутствовать.

Общая причина этих трудностей обусловлена тем, что обычный РГ-метод приводит к нефизическим особенностям у инвариантного заряда, которые нарушают аналитические свойства, вытекающие из общих принципов теории. Следовательно, требуется дальнейшая модификация пертурбативных разложений, такая, чтобы утерянные аналитические свойства были восстановлены. В работе [6] был предложен аналитический подход в КХД, объединяюший метод РГ и $Q^{2}$-аналитичность.

\section{4. АНАЛИТИЧЕСКАЯ ТЕОРИЯ ВОЗМУЩЕНИЙ}

В аналитическом подходе основным объектом является спектральная функция, которая входит в некоторое интегральное представление. Для двухточечных функций - это представление Челлена-Лемана, а для структурных функций неупругого лептон-адронного рассеяния таким представлением является представление Йоста-Лемана-Дайсона [1]. Спектральная функция рассматриваемых здесь объектов, таких как коррелятор или соответствующая ему $D$-функция, может быть вычислена по теории возмущений с использованием ряда (20) как "затравочного" 2),

$$
d_{\mathrm{pt}}\left(Q^{2}\right)=a_{\mathrm{pt}}\left(Q^{2}\right)+d_{1} a_{\mathrm{pt}}^{2}\left(Q^{2}\right)+d_{2} a_{\mathrm{pt}}^{3}\left(Q^{2}\right) .
$$

В результате АТВ-выражение имеет вид нестепенного разложения

$$
d_{\mathrm{an}}\left(Q^{2}\right)=a_{\mathrm{an}}\left(Q^{2}\right)+d_{1} \delta_{\mathrm{an}}^{(2)}\left(Q^{2}\right)+d_{2} \delta_{\mathrm{an}}^{(3)}\left(Q^{2}\right)
$$

где коэффициенты разложения $d_{1}$ и $d_{2}$ те же, что и в (22). Эти коэффициенты зависят от выбора схемы перенормировки и числа активных кварков $n_{f}$. В $\overline{\mathrm{MS}}$-схеме для $n_{f}=3$ они соответственно равны $d_{1}^{\overline{\mathrm{MS}}}=1.6398$ и $d_{2}^{\overline{\mathrm{MS}}}=6.3710$ [39]. Аналитические функции $\delta_{\mathrm{an}}^{(n)}\left(Q^{2}\right)$ в разложении (23) определяются через $\varrho_{n}(\sigma)=\operatorname{Im}\left[a_{\mathrm{pt}}^{n+1}(-\sigma-i \epsilon)\right][10],[40]$.

\footnotetext{
2) Здесь мы используем трехпетлевое приближение и опускаем черту надинвариантным зарядом $a=\alpha_{S} / \pi$. Индексы "pt" и “an" в дальнейшем соответствуют ТВ и АТВ.
} 
Инвариантные заряды $a_{\text {an }}\left(Q^{2}\right)$ и $a_{\mathrm{S}}(s)$, определенные соответственно в евклидовой и физической областях, выражаются через функцию $\varrho_{0}(\sigma)$ следуюшим образом:

$$
\begin{aligned}
a_{\mathrm{an}}\left(Q^{2}\right) & =\frac{1}{\pi} \int_{0}^{\infty} \frac{d \sigma}{\sigma+Q^{2}} \varrho_{0}(\sigma), \\
a_{\mathrm{S}}(s) & =\frac{1}{\pi} \int_{s}^{\infty} \frac{d \sigma}{\sigma} \varrho_{0}(\sigma) .
\end{aligned}
$$

Нередко подразумевается, что поведение инвариантного заряда в евклидовой $\left(Q^{2}>0\right)$ и в физической $\left(Q^{2}=-s<0\right)$ областях симметрично. Однако, как было показано в работе [41] на основе общих принципов теории, это утверждение несправедливо. Функции (24) и (25) имеют одинаковые инфракрасные предельные значения и одно и то же ультрафиолетовое поведение, но в промежуточной области они не совпадают.

В отличие от пертурбативного заряда $a_{\mathrm{pt}}\left(Q^{2}\right)$, функция $a_{\mathrm{an}}\left(Q^{2}\right)$ не обладает нефиизическими сингулярностями. В основном порядке инвариантные заряды в пространственноподобной и времениподобной областях записываются в виде [6], [8], [9]

$$
\begin{aligned}
a_{\mathrm{an}}^{(1)}\left(Q^{2}\right) & =\frac{1}{\beta_{0}}\left[\frac{1}{\ln \frac{Q^{2}}{\Lambda^{2}}}+\frac{\Lambda^{2}}{\Lambda^{2}-Q^{2}}\right], \\
a_{\mathrm{S}}^{(1)}(s) & =\frac{1}{\beta_{0}}\left[\frac{1}{2}-\frac{1}{\pi} \operatorname{arctg} \frac{\ln \frac{s}{\Lambda^{2}}}{\pi}\right] .
\end{aligned}
$$

Выражение (26) содержит обычный логарифмический член, который совпадает с пертурбативным выражением с призрачным полюсом при $Q^{2}=\Lambda^{2}$. Вклад этого полюса компенсируется вторым слагаемым в (26), которое имеет степенной характер. Будучи записанным в терминах исходного пертурбативного заряда $a_{\mathrm{pt}}$, это слагаемое содержит структуру типа $\exp \left(-1 / a_{\mathrm{pt}}\right)$, и его след исчезает в степенном пертурбативном разложении по $a_{\mathrm{pt}}$. Таким образом, невидимый в ТВ степенной по $Q^{2}$ вклад в аналитический инвариантный заряд (26) восстанавливается автоматически на основе свойства аналитичности. Регулярность инвариантного заряда во времениподобной области (27) вызвана другой причиной. Разложение функции (27) по сингулярному заряду $a_{\mathrm{pt}}\left(Q^{2}\right) \sim$ $1 / \ln \left(Q^{2} / \Lambda^{2}\right)$ демонстрирует появление $\pi^{2}$-членов.

В рамках АТВ чувствительность к выбору метода аппроксимации решения РГ-уравнения для пертурбативного заряда оказывается незначительной [10]. В данной работе при построении AТВ-разложений мы используем стандартную ТВ-параметризацию инвариантного заряда, что позволяет получить явное выражение для функции $a_{\mathrm{S}}(s)[42]$, являющееся трехпетлевым аналогом формулы (27). В более общем случае функции $a_{\mathrm{an}}\left(Q^{2}\right)$ и $a_{\mathrm{s}}(s)$ могут быть представлены в терминах функции Ламберта [43]-[45].

\section{5. $\tau$-РАСПАД}

Обычно при описании $\tau$-распада используют один из двух способов. Предложенное в [24] приближение приводит к представлению $\delta_{\tau}$ в виде разложения по степеням пертурбативного заряда $a_{\mathrm{pt}}$, взятого при $Q^{2}=M_{\tau}^{2}$. В этом случае трехпетлевое разложение имеет вид

$$
\delta_{\tau}^{\mathrm{I}}=a_{\mathrm{pt}}\left(M_{\tau}^{2}\right)+K_{1} a_{\mathrm{pt}}^{2}\left(M_{\tau}^{2}\right)+K_{2} a_{\mathrm{pt}}^{3}\left(M_{\tau}^{2}\right)
$$


где коэффициенты $K_{1}$ и $K_{2}$ в $\overline{\mathrm{MS}}$-схеме для трех активных кварков суть $K_{1}=5.2023$ и $K_{2}=26.366[22]$.

Подстановка функции $d_{\mathrm{pt}}$ в виде (22) в контурный интеграл (9) приводит к другому выражению, которое уже не является степенным разложением по $a_{\mathrm{pt}}[46]$,

$$
\delta_{\tau}^{\mathrm{II}}=A^{(1)}\left(M_{\tau}^{2}\right)+d_{1} A^{(2)}\left(M_{\tau}^{2}\right)+d_{2} A^{(2)}\left(M_{\tau}^{2}\right),
$$

где

$$
A^{(n)}\left(M_{\tau}^{2}\right)=\frac{1}{2 \pi i} \oint_{|z|=M_{\tau}^{2}} \frac{d z}{z}\left(1-\frac{z}{M_{\tau}^{2}}\right)^{3}\left(1+\frac{z}{M_{\tau}^{2}}\right) a_{\mathrm{pt}}^{n}(-z) .
$$

Оба способа описания $\tau$-распада широко используются в литературе. Несмотря на высокую точность экспериментально измеряемых величин $R_{\tau}[20]$, получаемое с их помошью значение инвариантного заряда имеет большую погрешность, доминируюший вклад в которую вносит теоретическая неопределенность КХД-вычислений. Так, например, стандартная обработка данных ALEPH коллаборации [18] приводит к значению $\alpha_{S}\left(M_{\tau}^{2}\right)=0.334 \pm 0.007_{\text {expt }} \pm 0.021_{\text {theor. }}$. Может показаться, что большая теоретическая погрешность связана с непертурбативным вкладом, который недостаточно хорошо известен. Однако оценки показывают, что полньй непертурбативный вклад пренебрежимо мал: $\delta_{\mathrm{npt}}=-0.003 \pm 0.004$ [18]. Поэтому главная неопределенность связана именно с использованием обсуждаемых здесь пертурбативных аппроксимаций.

Если метод вычислений сохраняет правильные аналитические свойства функции $d\left(Q^{2}\right)$, то как исходное представление (8), так и контурное (9) - эквивалентны. Например, при использовании аппроксимаций $d\left(Q^{2}\right)$ и $r(s)$ в виде исходных разложений (13) и (14), получаем, что формулы (8) и (9) приводят к одному и тому же результату:

$$
\begin{aligned}
\delta_{\tau}= & a_{\mathrm{pt}}\left(\mu^{2}\right) r_{1,0}+a_{\mathrm{pt}}^{2}\left(\mu^{2}\right)\left[\left(r_{2,0}-\frac{19}{12} r_{2,1}\right)+r_{2,1} \ln \left(\frac{M_{\tau}^{2}}{\mu^{2}}\right)\right]+ \\
& +a_{\mathrm{pt}}^{3}\left(\mu^{2}\right)\left[\left(r_{3,0}-\frac{19}{12} r_{3,1}+\frac{265}{72} r_{3,2}\right)+\right. \\
& \left.+\left(r_{3,1}-\frac{19}{6} r_{3,2}\right) \ln \left(\frac{M_{\tau}^{2}}{\mu^{2}}\right)+r_{3,2} \ln ^{2}\left(\frac{M_{\tau}^{2}}{\mu^{2}}\right)\right] .
\end{aligned}
$$

Теперь, если воспользоваться $\mu^{2}$-инвариантностью полного ряда и положить $\mu^{2}=M_{\tau}^{2}$, то можно прийти к разложению (28). Выражение (29) не может быть получено непротиворечивым образом. Действительно, контурное представление (30), в котором пертурбативный инвариантный заряд фигурирует под знаком интеграла по комплексной переменной, не является правомерным, поскольку нефизические сингулярности инвариантного заряда нарушают связь контурного представления с исходным выражением (8). На это явно указывает формула (21): для сохранения равенства между левой и правой частями пренебречь вкладом от $\Theta$-функции нельзя. Может показаться, что к контурному представлению (29) можно прийти другим способом. А именно, в качестве первого шага перейти к записи $\delta_{\tau}$ в виде контурного интеграла, используя при этом аппроксимации с параметром разложения $a\left(\mu^{2}\right)$. Затем выбрать (до вычисления контурного интеграла) 
величину $\mu^{2}$ переменным комплексным параметром, по которому выполняется интегрирование. Однако процедура перенормировки, в которой понятие о точке вычитания и возникает, такова, что значение $\mu^{2}$ не может быть произвольным. Комплексные значения этого параметра не гарантируют эрмитовость перенормированного лагранжиана. Таким образом, контурное представление $\delta_{\tau}$ в виде $(29)$ при использовании сингулярного инвариантного заряда не является самосогласованным.

В аналитическом подходе эквивалентность выражений (8) и (9) сохраняется. В терминах эффективной спектральной функции $\rho^{\text {eff }}(\sigma)=\rho_{0}(\sigma)+d_{1} \rho_{1}(\sigma)+d_{2} \rho_{2}(\sigma)$ получаем

$$
\delta_{\tau}=\frac{1}{\pi} \int_{0}^{\infty} \frac{d \sigma}{\sigma} \rho^{\mathrm{eff}}(\sigma)-\frac{1}{\pi} \int_{0}^{M_{\tau}^{2}} \frac{d \sigma}{\sigma}\left(1-\frac{\sigma}{M_{\tau}^{2}}\right)^{3}\left(1+\frac{\sigma}{M_{\tau}^{2}}\right) \rho^{\mathrm{eff}}(\sigma)
$$

\section{6. ЧИСЛЕННЫЕ РЕЗУЛЬТАТЫ}

АТВ-аппроксимации имеют не только правильные аналитические свойства, но и обладают петлевой стабильностью и малой чувствительностью к выбору ренормализационного предписания. Стабильность АТВ по отношению к высшим петлевым поправкам демонстрируется на рис. 1 , на котором показано поведение отношения $R_{\tau}$ в одно-, двух- и трехпетлевом приближениях в зависимости от значения инвариантного заряда на масштабе массы $\tau$-лептона. Здесь же приведены результаты ТВ, полученные из (28). Изображенный на рисунке коридор ошибок соответствует экспериментальному значению $R_{\tau}^{\text {expt }}=3.646 \pm 0.022[47]$.

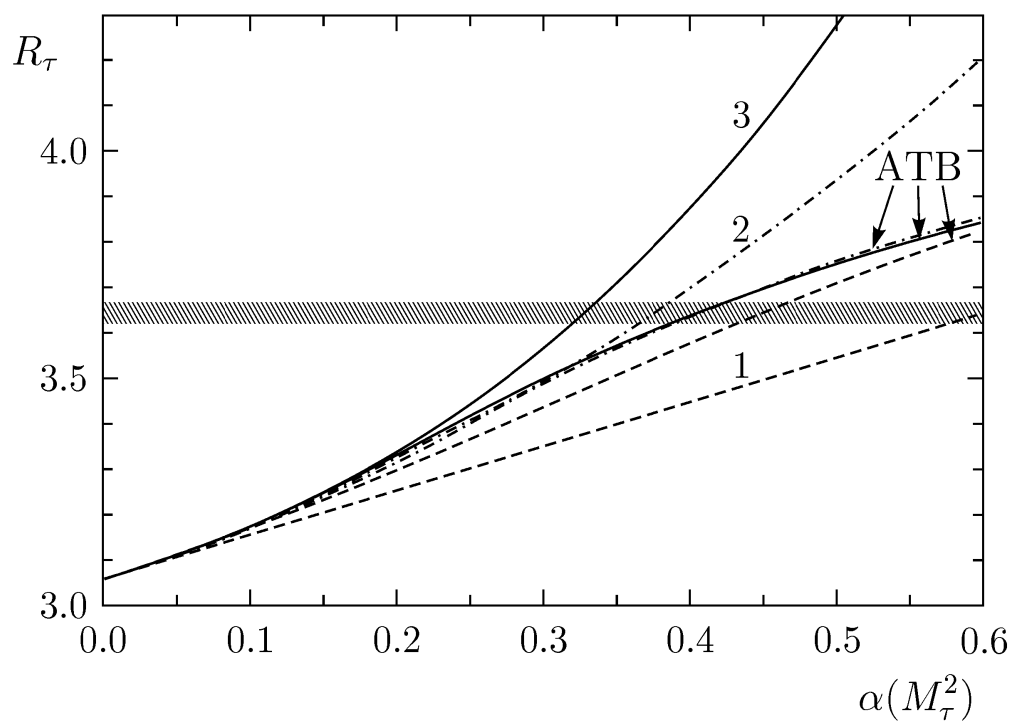

Рис. 1. Поведение $R_{\tau}$ в однопетлевом (штриховые линии), двухпетлевом (штрихпунктир) и трехпетлевом (сплошные кривые) приближениях для ТВ- и ATB-аппроксимаций в MS-схеме. Цифры над кривыми соответствуют порядку ТВ-приближения, стрелки указывают на АТВ-результат. 
Отметим, что аналогичный анализ инклюзивного $\tau$-распада с использованием контурного ТВ-представления и аналитического подхода представлен в работе [48]. В этой работе в разделе, посвяшенном аналитическому подходу, обсуждаются значения $\alpha_{\text {an }}$, лежащие в интервале $1.5 \div 2.0$, которые, по мнению авторов, следует принять для соответствия с экспериментальными данными. Это утверждение не согласуется с результатом АТВ, показанным на рис. 1. Как видно из этого рисунка, для согласия с экспериментом требуется значение $\alpha_{\text {an }} \simeq 0.4$. Здесь уместно подчеркнуть, что в аналитическом подходе величина инвариантного заряда $\alpha_{\text {an }}$ ограничена сверху и не может превышать предельного инфракрасного значения $\pi / \beta_{0} \simeq 1.4$ [6]. Мы согласны с авторами [48] в том, что суммирование $\pi^{2}$-членов важно в области промежуточных энергий ${ }^{3)}$, и в том, что использование выражения (27), суммируюшего $\pi^{2}$-вклады, вместо асимптотического $\sim 1 / \ln \left(s / \Lambda^{2}\right)$ является предпочтительным. При этом важно отметить, что выражение (27) приводит в евклидовой области к аналитическому заряду (26). Таким образом, формулы типа (26) и (27) взаимосвязаны, и аналитический подход осушествляет последовательное совместное их рассмотрение. Иными словами, суммирование $\pi^{2}$-вкладов дает регулярную функцию и приводит к появлению степенных (нелогарифмических) вкладов в пространственноподобной области, которые компенсируют нефизические особенности обычных логарифмических слагаемых.

Как известно, важным источником теоретической неопределенности может служить зависимость результатов от выбора ренормализационного предписания. В КХД такая неопределенность тем больше, чем меньше характерный энергетический масштаб процесса. Схемная зависимость ТВ-аппроксимаций для инклюзивного распада $\tau$-лептона исследовалась в ряде работ (см., например, [32], [40], [49]). Здесь мы обсудим этот вопрос для $D$-функции, информация о которой также может быть получена из богатого экспериментального материала, накопленного в измерениях адронных мод $\tau$-распада [42], [50], [51].

Каких-либо аргументов общего характера, которые позволяли бы с самого начала отдать предпочтение той или иной схеме перенормировок, не сушествует. Тем не менее можно определить некоторый класс схем, воспользовавшись критерием, предложенным в работе [52]. Согласно этому критерию следует ограничиться теми схемами, для которых сокращения меж ду слагаемыми во втором схемном инварианте $\omega_{2}=c_{2}+d_{2}-c_{1} d_{1}-$ $d_{1}^{2}$, построенном из коэффициентов $d_{1}, d_{2}(22)$ и $c_{1}, c_{2}$, которые определяются двухпетлевым и схемно-зависимым трехпетлевым коэффициентами $\beta$-функции, не слишком велики. Количественно такой критерий можно соотнести с величиной индекса сокрашений

$$
C=\frac{\left|c_{2}\right|+\left|d_{2}\right|+c_{1}\left|d_{1}\right|+d_{1}^{2}}{\left|\omega_{2}\right|}
$$

Выбирая некоторое максимальное значение индекса сокращений $C_{\max }$, которое определит границу для класса допустимых схем, и перебирая схемы с индексом $C \leqslant C_{\max }$,

\footnotetext{
${ }^{3)}$ Более того, корректное обращение с $\pi^{2}$-членами при обработке экспериментальных данных во времениподобной области оказывается также важным и в традиционно считающейся асимптотической области порядка нескольких десятков ГэВ и выше [15].
} 
можно изучить стабильность получаемых результатов. Сравнительно узкий класс "разрешенных" схем возникает, если значение $C_{\max }$ выбрать соответствуюшим PMS-оптимизации. Зависимость трехпетлевого вклада в $D$-функцию от выбора ренормализационного предписания приведена на рис. 2.

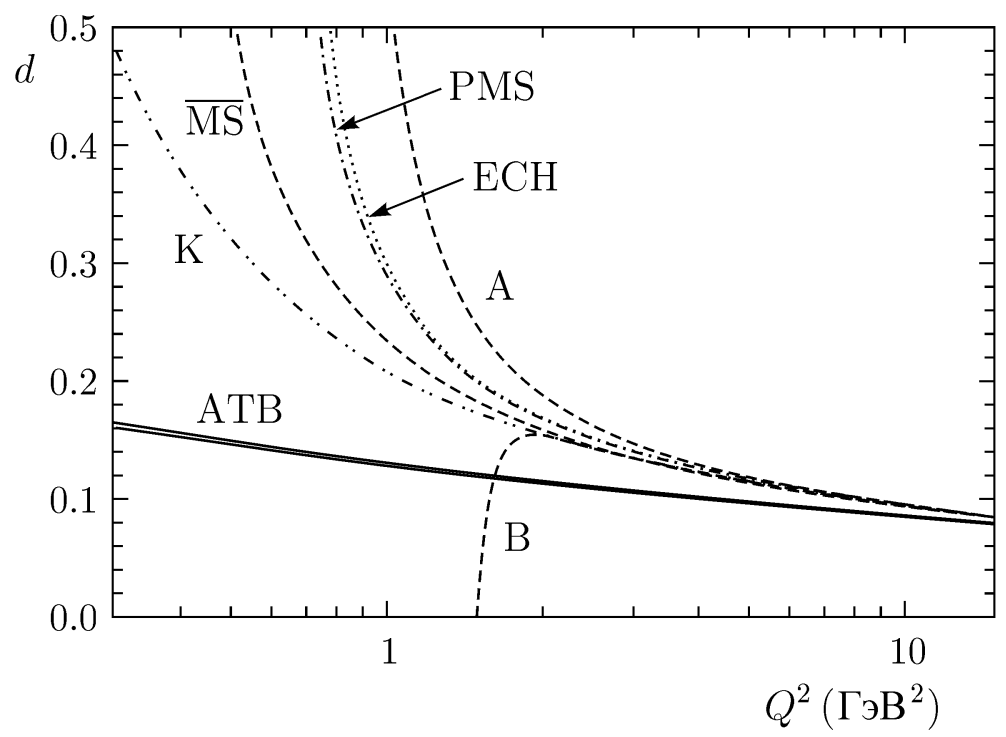

Рис. 2. Поведение функции $d\left(Q^{2}\right)$ в различных схемах перенормировки в ТВ и АТВ.

АТВ соответствуют сплошные линии, которые практически сливаются в одну кривую.

Расчеты выполнены в схемах A, B, PMS, близких друг к другу в смысле индекса сокрашений $\left(C_{\mathrm{A}} \simeq C_{\mathrm{B}} \simeq C_{\mathrm{PMS}} \simeq 2\right)$, и ЕСН-схеме, которая также входит в класс очерченных вьше "разрешенных" схем $\left(C_{\mathrm{ECH}}=1\right)$. Кроме того, приведены результаты для шшроко используемых $\overline{\mathrm{MS}}$-схемы $\left(C_{\overline{\mathrm{MS}}} \simeq 3,1\right)$ и $\mathrm{K}$-схемы $\left(C_{\mathrm{K}} \simeq 5,3\right)$, интересной тем, что в ней имеется инфракрасно-стабильная точка [53]. Все изображенные на рис. 2 кривые отвечают одному и тому же значению масштабного параметра, заданного в $\overline{\mathrm{MS}}$-схеме, $\Lambda \frac{(3)}{\mathrm{MS}}=370 \mathrm{MэB} \mathrm{[18].} \mathrm{Из} \mathrm{рис.} 2$ видно, что кривые для $d\left(Q^{2}\right)$, полученные в ТВ для различных схем перенормировки, значительно различаются при значении $Q^{2} \simeq$ $(1 \div 2)$ ГэВ ${ }^{2}$. Результаты АТВ, полученные в тех же самых схемах, практически совпадают и на графике сливаются в одну кривую.

\section{7. ЗАКЛЮЧЕНИЕ}

В работе проведен сравнительный анализ достоинств и недостатков различных форм пертурбативного разложения как с обших позиший, так и в контексте применения к инклюзивному распаду $\tau$-лептона. Приведены аргументы в пользу АТВ, которая не только согласуется с обшими принципами теории, но и обладает рядом достоинств практического характера. В аналитическом подходе два способа описания процесса инклюзивного распада $\tau$-лептона в терминах времениподобных или евклидовых переменных эквивалентны. В АТВ зависимость результатов от выбора ренормализационного предписа- 
ния существенным образом снижена и можно говорить о практической независимости трехпетлевых выражений от схемы перенормировки. Таким образом, расчеты на основе АТВ значительно снижают теоретическую неопределенность результатов. Следовательно, ее использование в качестве пертурбативной компоненты повышает надежность информации о параметрах КХД, получаемой из экспериментальных данных по $\tau$-распаду, которые известны с высокой точностью.

Благодарности. Автор благодарит А.Н. Сисакяна и Д. В. Ширкова за интерес к работе и поддержку, а также С. В. Михайлова и И. Л. Соловцова за полезные обсуждения полученных результатов. Работа выполнена при поддержке РФФИ (грант № 02-0100601).

\section{Список литературы}

[1] Н. Н. Боголюбов, Д. В. Ширков. Введение в теорию квантованных полей. М.: Наука, 1986.

[2] E. C. G. Stukelberg, A. Peterman. Helv. Phys. Acta. 1953. V. 26. P. 499.

[3] M. Gell-Mann, F.E. Low. Phys. Rev. 1954. V. 95. P. 1300.

[4] Н. Н. Боголюбов, Д. В. Ширков. ДАН СССР. 1955. Т. 103. С. 203; ЖЭТФ. 1956. Т. 30. C. 77 ; N. N. Bogoluibov, D. V. Shirkov. Nuovo Cimento. 1956. V. 3. P. 845.

[5] Н. Н. Боголюбов, А. А. Логунов, Д. В. Ширков. ЖЭТФ. 1959. Т. 37. С. 805.

[6] D. V. Shirkov, I. L. Solovtsov. JINR Rapid Comm. 1996. V. 2. P. 5; Phys. Rev. Lett. 1997. V. 79. P. 1209.

[7] И. Л. Соловцов, Д. В. Ширков. ТМФ. 1999. Т. 120. № 3. С. 482.

[8] K. A. Milton, I. L. Solovtsov. Phys. Rev. D. 1997. V. 55. P. 5295.

[9] K. A. Milton, O. P. Solovtsova. Phys. Rev. D. 1998. V. 57. P. 5402.

[10] K. A. Milton, I. L. Solovtsov, O. P. Solovtsova. Phys. Lett. B. 1997. V. 415. P. 104.

[11] I. L. Solovtsov, D. V. Shirkov. Phys. Lett. B. 1998. V. 442. P. 344.

[12] Д. В. Ширков. ТМФ. 1998. Т. 119. № 1. С. 55; hep-th/9810246.

[13] K. A. Milton, I. L. Solovtsov, O. P. Solovtsova. Phys. Rev. D. 1999. V. 60. P. 016001.

[14] I. L. Solovtsov. Part. Nucl. Lett. 2000. V. 4. P. 10.

[15] Д. В. Ширков. ТМФ. 2001. Т. 127. № 1. С. 3.

[16] E. V. Shuryak, A.I. Vainshtein. Nucl. Phys. B. 1982. V. 199. P. 451.

[17] V. M. Braun, A. V. Kolesnichenko. Nucl. Phys. B. 1987. V. 283. P. 723.

[18] The ALEPH Collaboration, R. Barate et al. Eur. Phys. J. C. 1998. V. 4. P. 409.

[19] The OPAL Collaboration, K. Ackerstaff et al. Eur. Phys. J. C. 1999. V. 7. P. 571

[20] S. H. Robertson. Charged current review. In: TAU 2000. Nucl. Phys. B. (Proc. Suppl.). Eds. R. J. Sobie, J. M. Roney, 2001. V. 98. P. 67.

[21] Y.S. Tsai. Phys. Rev. D. 1971. V. 4. P. 2821.

[22] E. Braaten, S. Narison, A. Pich. Nucl. Phys. B. 1992. V. 373. P. 581.

[23] S. L. Adler. Phys. Rev. D. 1974. V. 10. P. 3714.

[24] E. Braaten. Phys. Rev. Lett. 1988. V. 60. P. 1606; Phys. Rev. D. 1989. V. 39. P. 1458.

[25] B. Schrempp, F. Schrempp. Z. Phys. C. 1980. V. 6. P. 7.

[26] A. V. Radyushkin. JINR Rapid Commun. 1996. V. 78. P. 96; hep-ph/9907228.

[27] N. V. Krasnikov, A. A. Pivovarov. Phys. Lett. B. 1982. V. 116. P. 168.

[28] J. D. Bjorken. Two topics in QCD. Preprint SLAC-PUB-5103, 1989; In: Proc. Cargese Summer Institute in Particle Physics. NATO Adv. Study Inst. Ser. B. V. 223. Eds. M. Levy et al. N.Y.: Plenum, 1990.

[29] D. V. Shirkov. Eur. Phys. J. C. 2001. V. 22. P. 331; hep-ph/0107282.

[30] K. G. Chetyrkin. Phys. Lett. B. 1997. V. 390. P. 309.

[31] P. M. Stevenson. Phys. Lett. B. 1981. V. 100. P. 61; Phys. Rev. D. 1981. V. 23. P. 2916. 
[32] J. Chyla, A. L. Kataev, S. G. Larin. Phys. Lett. B. 1991. V. 267. P. 269.

[33] A. C. Mattingly, P. M. Stevenson. Phys. Rev. Lett. 1992. V. 69. P. 1320; Phys. Rev. D. 1994. V. 49. P. 437.

[34] G. Grunberg. Phys. Lett. B. 1980. V. 95. P. 70; Phys. Rev. D. 1984. V. 29. P. 2315

[35] A. Dhar, V. Gupta. Phys. Rev. D. 1984. V. 29. P. 2822.

[36] S. G. Gorishny, A.L. Kataev, S. A. Larin, L.R. Surguladze. Phys. Rev. D. 1991. V. 43. P. 1633.

[37] K. A. Milton, I. L. Solovtsov. Infrared fixed point of the analytic running coupling. In: Proc. XIV Int. Seminar on High Energy Physics Problems: Relativistic Nuclear Physics and Quantum Chromodynamics. Eds. A. M. Baldin, V.V. Burov. Dubna: JINR, 2000. V. I. P. 47.

[38] J. A. M. Vermaseren, S. A. Larin, T. van Ritbergen. Phys. Lett. B. 1997. V. 405. P. 327.

[39] S. G. Gorishny, A. L. Kataev, S. A. Larin. Phys. Lett. B. 1991. V. 259. P. 144.

[40] K. A. Milton, I. L. Solovtsov, O. P. Solovtsova, V. I. Yasnov. Eur. Phys. J. C. 2000. V. 14. P. 495.

[41] K. A. Milton, I. L. Solovtsov. Phys. Rev. D. 1999. V. 59. P. 107701.

[42] K. A. Milton, I. L. Solovtsov, O. P. Solovtsova. Phys. Rev. D. 2001. V. 64. P. 016005.

[43] E. Gardi, G. Grunberg, M. Karliner. JHEP. 1998. V. 07. P. 007.

[44] B. A. Magradze. Int. J. Mod. Phys. A. 2000. V. 15. P. 2715.

[45] D. S. Kourashev, B. A. Magradze. Explicit expressions for Euclidean and Minkowskian QCD observables in analytic perturbation theory. hep-ph/0104142.

[46] F. Le Diberder, A. Pich. Phys. Lett. B. 1992. V. 286. P. 147.

[47] D. E. Groom et al. Particle Data Group. Eur. Phys. J. C. 2000. V. 15. P. 1.

[48] B. V. Geshkenbein, B.L. Ioffe, K. N. Zyablyuk. Phys. Rev. D. 2001. V. 64. P. 093009; hep-ph/0104048.

[49] P. A. Raczka, A. Szymacha. Z. Phys. C. 1996. V. 70. P. 125.

[50] S. Peris, M. Perrottet, E. de Rafael. JHEP. 1998. V. 05. P. 011.

[51] А. Н. Сисакян, И. Л. Соловцов, О. П. Соловцова. Письма в ЖЭТФ. 2001. Т. 73. С. 186.

[52] P. A. Raczka. Z. Phys. C. 1995. V. 65. P. 481.

[53] N. V. Krasnikov, A. A. Pivovarov. Mod. Phys. Lett. A. 1996. V. 11. P. 835.

Поступила в редакцию 12.IX.2001 г., после доработки 17.VI.2002 г. 